\title{
A volume maximizing canonical surface in 3-space
}

\author{
Ingrid C. Bauer and Fabrizio Catanese
}

\begin{abstract}
Answering a question posed by Enriques, we construct a minimal smooth algebraic surface $S$ of general type over the complex numbers with $K^{2}=45$ and $p_{g}=4$, and with birational canonical map. The canonical system $\left|K_{S}\right|$ has a fixed part and the degree of the canonical image is 19. The surface we construct is rigid, $S$ is indeed a ball quotient. It is obtained as an Abelian covering of the plane branched over an arrangement of lines already considered by Hirzebruch, and it is the first such example which is regular $(q=0)$.
\end{abstract}

Mathematics Subject Classification (2000). 14J25, 14J29, 14J80.

Keywords. Regular ball quotients, configurations of lines.

\section{Introduction}

At the onset of surface theory surfaces in 3-space, and especially canonical surfaces in 3-space, occupied a central role.

In particular, this study led to the famous Noether inequality $K^{2} \geq 2 p_{g}-4$, while Castelnuovo observed that if the canonical map of a minimal smooth surface $S$ is birational (obviously then $p_{g} \geq 4$ ) the inequality $K^{2} \geq 3 p_{g}-7$ must hold true.

These are the lower bounds for surface geography, but upper bounds played a decisive role in the investigations of the last 30 years, leading to the so called BogomolovMiyaoka-Yau inequality

$$
K^{2} \leq 9 \chi:=9\left(p_{g}-q+1\right)
$$

\section{(cf. [BPV], Chapter VII, Section 4).}

For instance, the BMY inequality gives a hypothetical upper bound for a question raised by F. Enriques (cf. [Enr], Chapter VIII, p. 284).

Problem. Which are the possible values of $K^{2}$, in particular which is the highest possible value of $K^{2}$ for minimal surfaces with geometric genus $p_{g}=4$ having a birational canonical map (so-called simple canonical surfaces)? 
In fact, Enriques even conjectured that the highest possible value for $K^{2}$ should be 24 , based on the conjecture that the expected number of moduli should be strictly positive. The second author showed in [Cat2] that this bound does not hold true, constructing simple canonical surfaces with geometric genus $p_{g}=4$ and $11 \leq$ $K^{2} \leq 28$ (in these examples $K^{2}$ equals the 'canonical degree', i.e., the degree of the canonical image). This bound was improved by C. Liedtke (cf. [Lie]) who showed the existence of a simple canonical surface with $p_{g}=4$ and $K^{2}=31$ (and canonical degree 12).

Simple canonical surfaces have $K^{2} \geq 5$, and for $5 \leq K^{2} \leq 7$ they were constructed by Enriques, Franchetta, Kodaira, Maxwell, and for $6 \leq \bar{K}^{2} \leq 16$ by Burniat, while Ciliberto was able to show for $5 \leq K^{2} \leq 10$ the existence of simple canonical surfaces with ordinary singularities (cf. [Enr], [Fran], [Max], [Kod], [Bur], [Cil]).

If we try to go up with $K^{2}$, the BMY inequality tells us that $K^{2} \leq 45$, and that, if equality holds, then necessarily $S$ is regular $(q(S)=0)$.

The main result of this paper is the following

Main Theorem. There exists a minimal smooth algebraic surface $S$ of general type over the complex numbers with $K^{2}=45$ and $p_{g}=4$, and with birational canonical map. $S$ is rigid, the canonical system $\left|K_{S}\right|$ has a fixed part and the degree of the canonical image is 19.

More precisely, consider the smooth $(\mathbb{Z} / 5)^{2}$-Galois covers of $\mathbb{P}_{\mathbb{C}}^{2}$ branched on a complete quadrangle. These are 4 ball quotients $S_{i}, i=1,2,3,4$, with $\chi\left(S_{i}\right)=5$, $K_{S_{i}}^{2}=45$ and $q\left(S_{3}\right)=0, q\left(S_{i}\right)=2$ for $i=1,2,4$.

For $S_{3}$ the canonical system $\left|K_{S_{3}}\right|$ has a fixed part $\Psi$, the movable part $|M|:=$ $\left|K_{S_{3}}-\Psi\right|$ has 5 base points, and the canonical map $\phi_{K}: S_{3} \rightarrow \Sigma$ is birational onto a surface $\Sigma$ of degree 19.

Finally, the first homology groups are as follows:

$$
H_{1}\left(S_{3}, \mathbb{Z}\right) \cong(\mathbb{Z} / 5)^{4}, \quad H_{1}\left(S_{i}, \mathbb{Z}\right) \cong(\mathbb{Z} / 5)^{2} \oplus \mathbb{Z}^{4}, \quad i \neq 3 .
$$

The rigidity of $S$ is due to the fact that, by Yau's proof of the inequality $K^{2} \leq 9 \chi$, it follows (cf. also [Miy]) that $K^{2}=9 \chi$ if and only if the universal covering of $S$ is the 2-dimensional complex ball $\mathfrak{B}_{2}$.

It was for long time extremely hard to give direct algebro geometric constructions of such ball quotients, until a breakthrough came via the explicit constructions by Hirzebruch as Kummer coverings of the complex projective plane branched in a configuration of lines ([Hir]). These examples were extended and generalized in the book $[\mathrm{BHH}]$, which amply describes three examples of such (compact) ball quotients. The configurations occurring are quite classical: a complete quadrangle, the Hesse configuration and the dual Hesse configuration. Even if it is possible to determine the numerical data which a configuration has to fulfill in order to give rise to a ball quotient, it is less easy to compute the holomorphic invariants. 
In fact, already the determination of the irregularities $q$ of the Hirzebruch examples and of some étale quotients of them required further work by M.-N. Ishida (cf. [Ish1], [Ish2]), but no regular examples were indeed found (except Mumford's fake projective plane, whose construction however was not so explicit as Hirzebruch's one, see [Mum]).

The example of $[\mathrm{BHH}]$ we are interested in here is the $(\mathbb{Z} / 5 \mathbb{Z})^{5}$-covering $\hat{S}$ of $\mathbb{P}^{2}$ branched exactly in a complete quadrangle $\Delta$. This surface has the invariants $K^{2}=$ $45 \cdot 125$ and $\chi=5 \cdot 125$. It is clear that an étale $(\mathbb{Z} / 5 \mathbb{Z})^{3}$ quotient or, equivalently, a smooth $(\mathbb{Z} / 5 \mathbb{Z})^{2}$ covering of $\mathbb{P}^{2}$ branched exactly in a complete quadrangle has the invariants $K^{2}=45$ and $\chi=5$. Since, as we observed, $\chi=p_{g}-q+1$, we have to produce an example of a surface $S$ which is regular (i.e., $q=0$ ) in order to get the desired example of a surface with $K^{2}=45$ and $p_{g}=4$. In fact, we will show that up to isomorphisms there are exactly four smooth surfaces with $K^{2}=45, \chi=5$, obtained as $(\mathbb{Z} / 5 \mathbb{Z})^{2}$ coverings of $\mathbb{P}^{2}$ branched exactly in a complete quadrangle: but only one of them is regular (has $q=0$ ).

The main ingredient of our investigation is the theory of Abelian Galois coverings, developed by Pardini (cf. [Par]), but apparently not sufficiently known. Since the treatment by Pardini is very algebraic, and at some points not so explicit, we devote Section 1 to explain the structure theorem for such Abelian coverings, and especially the relation occurring between the topological data (which allow to construct the examples) and the explicit determination of the character sheaves (or eigensheaves) of the covering (these determine not only the topological but also the holomorphic invariants of the constructed surface).

Sections 2 and 3 are devoted to the construction of our surfaces, and to the investigation of the symmetries of our construction. This study allows us to classify all the examples up to isomorphisms.

Section 4 is devoted to the study of the canonical map of $S_{3}$, while in Section 5 we give a description of the fundamental group $\pi_{1}(S)$ of our surfaces, from which one can calculate the first homology group $H_{1}(S, \mathbb{Z})$.

\section{Abelian covers}

In this section we shall recall the structure theorem for normal Abelian Galois ramified coverings. We shall give a more direct presentation than the one in the original paper by R. Pardini (cf. [Par]). This will turn out to be more suitable for our purposes.

Let $X, Y$ be normal projective varieties, assume $Y$ to be smooth and let $\pi: X \rightarrow Y$ be a finite Galois cover with Abelian Galois group $G$. By the theorem on the purity of the branch locus the critical set of $\pi$ is a divisor $R$, the ramification divisor, whose image $D:=\pi(R)$ is called the branch divisor. In the case where also $X$ is smooth we have the following result (cf. [Cat1], Proposition 1.1). 
Proposition 1.1. If $X$ is smooth, then $R$ is a normal crossing divisor with smooth components. Moreover, if $x \in X$, then the stabilizer of $x$ is the direct sum of the stabilizers of the components of $R$ passing through $x$ and these last groups are cyclic.

We may assume without loss of generality, and will assume in the following, that $Y$ is smooth and $D$ is a normal crossing divisor.

We remark that $\pi$ factors canonically as

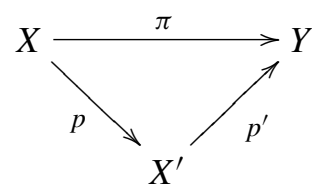

where $X^{\prime}$ is maximal such that $p^{\prime}: X^{\prime} \rightarrow Y$ is unramified. In fact, one takes $X^{\prime}:=$ $X / G^{\prime}$, where $G^{\prime}$ is the subgroup of $G$ generated by the stabilizers $G_{x}$ of points $x \in X$.

Definition 1.2. $\pi$ is called totally ramified iff $p^{\prime}$ is an isomorphism (i.e., $G=G^{\prime}$ ).

Observe that $\pi$ is necessarily totally ramified if $Y$ has a trivial algebraic fundamental group.

Now, $\pi$ is determined by the surjective homomorphism $\phi: \pi_{1}(Y-D) \rightarrow G$, which factors through $\varphi: H_{1}(Y-D, \mathbb{Z}) \rightarrow G$, since $G$ is assumed to be Abelian.

We denote by $G^{*}$ the group of characters of $G$, and we shall use the additive notation for the group operation in $G^{*}$. Recall that $\pi$ is flat (for this it suffices that $Y$ is smooth and $X$ is normal) and that the action of $G$ induces a splitting of the direct image of $\mathcal{O}_{X}$ into eigensheaves

$$
\pi_{*} \mathcal{O}_{X}=\bigoplus_{\chi \in G^{*}} \mathcal{L}_{\chi}^{-1},
$$

where $G$ acts on the invertible sheaf $\mathcal{L}_{\chi}^{-1}$ via the character $\chi$.

Note that $\mathcal{L}_{1} \cong \mathcal{O}_{Y}$ and denote by $L_{\chi}$ a divisor associated to the eigensheaf $\mathcal{L}_{\chi}$ (thus $\mathscr{L}_{\chi} \cong \mathcal{O}\left(L_{\chi}\right)$ ).

We shall show how

(1) one calculates $H_{1}(Y-D, \mathbb{Z})$;

(2) one calculates the character sheaves $\mathcal{L}_{\chi}=\mathcal{O}\left(L_{\chi}\right)$ in terms of the surjective homomorphism $\varphi: H_{1}(Y-D, \mathbb{Z}) \rightarrow G$.

Consider the exact sequence

$$
0 \rightarrow K \rightarrow H_{1}(Y-D, \mathbb{Z}) \rightarrow H_{1}(Y, \mathbb{Z}) \rightarrow 0 .
$$

Remark 1.3. If $\pi$ is totally ramified, $\varphi \mid K: K \rightarrow G$ is surjective. 
Vol. 83 (2008)

Following the arguments in [Cat1] we obtain

\section{Proposition 1.4.}

$$
K=\operatorname{ker}\left(H_{1}(Y-D) \rightarrow H_{1}(Y)\right)=\operatorname{coker}\left(r: H^{2 n-2}(Y) \rightarrow H^{2 n-2}(D)\right) .
$$

In particular, if $H_{1}(Y, \mathbb{Z})=0$, then

$$
H_{1}(Y-D, \mathbb{Z})=\operatorname{coker}\left(r: H^{2 n-2}(Y) \rightarrow H^{2 n-2}(D)\right) .
$$

Proof. Let $V$ be an open tubular neighbourhood of $D$ and denote by $\partial V$ its boundary. Then we have the exact sequence

$$
\cdots \rightarrow H^{2 n-2}(Y) \rightarrow H^{2 n-2}(D) \rightarrow H^{2 n-1}(Y, \bar{V}) \rightarrow H^{2 n-1}(Y) \rightarrow \cdots .
$$

Observing that $H^{2 n-1}(Y, \bar{V}) \cong H_{1}(Y-D, \mathbb{Z})$, we see that

$$
\begin{aligned}
K & =\operatorname{ker}\left(H_{1}(Y-D, \mathbb{Z}) \rightarrow H_{1}(Y, \mathbb{Z})\right) \\
& \cong \operatorname{coker}\left(r: H^{2 n-2}(Y) \rightarrow H^{2 n-2}(D) \cong \bigoplus_{i=1}^{k}\left[D_{i}\right] \mathbb{Z}\right) .
\end{aligned}
$$

Remark 1.5. Applying $\operatorname{Hom}_{\mathbb{Z}}(\cdot, G)$ to the short exact sequence (1) above we get

$$
\begin{aligned}
0 & \rightarrow \operatorname{Hom}\left(H_{1}(Y, \mathbb{Z}), G\right) \rightarrow \operatorname{Hom}\left(H_{1}(Y-D, \mathbb{Z}), G\right) \rightarrow \operatorname{Hom}(K, G) \\
& \rightarrow \operatorname{Ext}^{1}\left(H_{1}(Y, \mathbb{Z}), G\right) \rightarrow \operatorname{Ext}^{1}\left(H_{1}(Y-D, \mathbb{Z}), G\right) \rightarrow \operatorname{Ext}^{1}(K, G) \rightarrow 0 .
\end{aligned}
$$

Hence an Abelian covering of $Y$ ramified in $D$ is uniquely determined by a surjective morphism $\varphi: K \rightarrow G$ if and only if $\operatorname{Hom}\left(H_{1}(Y), G\right)=0$ and $\operatorname{Ext}^{1}\left(H_{1}(Y), G\right) \rightarrow$ $\operatorname{Ext}^{1}\left(H_{1}(Y-D), G\right)$ is injective. This happens, for instance, if $H_{1}(Y, \mathbb{Z})=0$, or more generally if $H_{1}(Y, \mathbb{Z})$ is a finite group whose exponent is relatively prime to the exponent of $G$.

Let us determine the character sheaves of the Abelian covering determined by $\varphi: H_{1}(Y-D, \mathbb{Z}) \rightarrow G$.

Let $\chi \in G^{*}$ be a character of $G$, i.e., $\chi: G \rightarrow C \subset \mathbb{C}^{*}$, where $C$ is cyclic. Then $\chi$ induces a surjective morphism $\chi \circ \varphi: H_{1}(Y-D, \mathbb{Z}) \rightarrow C$, whence a factorization of $\pi$ as

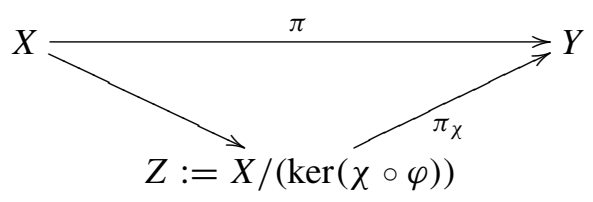

where $\pi_{\chi}: Z \rightarrow Y$ is a cyclic covering with group $C$. 
Remark 1.6. $\mathcal{L}_{\chi}(Z)=\mathcal{L}_{\chi}(X)$, and we are reduced to calculate the character sheaves for cyclic coverings.

Write $D=\bigcup_{i=1}^{k} D_{i}$ as a union of smooth irreducible components and denote by $\delta_{i}$ the image of a small loop around $D_{i}$ in $H_{1}(Y-D, \mathbb{Z})$.

Let $d$ be the order of $C$ and let us identify $C$ with $\mathbb{Z} / d$; then we have the well known formula (cf. the proof of Proposition 4.5 of [Ba-Ca])

$$
\mathcal{O}_{Y}\left(d L_{\chi}\right) \cong \mathcal{O}_{Y}\left(\sum_{i=1}^{k}(\chi \circ \varphi)\left(\delta_{i}\right) D_{i}\right)
$$

Remark 1.7. We remark that the above linear equivalence

$$
d L_{\chi} \equiv \sum_{i=1}^{k}(\chi \circ \varphi)\left(\delta_{i}\right) D_{i},
$$

depends only on $\chi \circ(\varphi \mid K)$ and does not uniquely determine the character sheaf $\mathcal{L}_{\chi}$. In fact, if $\mathcal{L}_{\chi} \in \operatorname{Pic}(Y)$ satisfies the above equation, then also $\mathcal{L}_{\chi} \otimes \eta$ does, for each $d$ torsion sheaf $\eta \in \operatorname{Pic}(Y)$. If $\eta$ corresponds to an element $\alpha \in \operatorname{Hom}\left(H_{1}(Y, \mathbb{Z}), \mathbb{Z} / d \mathbb{Z}\right)$, then $\mathcal{L}_{\chi} \otimes \eta$ is the character sheaf of the cyclic covering corresponding to $\chi \circ \varphi+\alpha \circ p$, where $p: H_{1}(Y-D, \mathbb{Z}) \rightarrow H_{1}(Y, \mathbb{Z})$. Clearly $(\chi \circ \varphi+\alpha \circ p)|K=(\chi \circ \varphi)| K$.

The observation that $p$ is surjective shows however once more that $\mathcal{L}_{\chi}$ is uniquely determined by $\phi$.

Choosing a fixed system of representatives of $\mathbb{Z} / d \mathbb{Z}$, e.g., $\mathbb{Z} / d \mathbb{Z}=\{0, \ldots, d-1\}$, we get then a unique representation

$$
d L_{\chi} \equiv \sum_{i=1}^{k}(\chi \circ \varphi)\left(\delta_{i}\right) D_{i}=\sum_{i=1}^{k} \Delta_{i} D_{i}, 0 \leq \Delta_{i} \leq d-1 .
$$

We will now use the above approach in order to write explicit equations for $X$ as a subvariety in the geometric vector bundle corresponding to the locally free sheaf $\bigoplus_{\chi \in G^{*} \backslash\{1\}} \mathcal{L}_{\chi}$.

Remark 1.8. Let $\chi: G \rightarrow C \cong \mathbb{Z} / d, \chi^{\prime}: G \rightarrow C \cong \mathbb{Z} / d^{\prime}$ be two characters of $G$. Then $\operatorname{ord}\left(\chi+\chi^{\prime}\right)=1$.c.m. $\left(d, d^{\prime}\right)=: M$. Write $M$ as $M=\lambda \cdot d=\lambda^{\prime} \cdot d^{\prime}$. Consider the linear equivalences

$$
\begin{aligned}
d L_{\chi} & \equiv \sum_{i=1}^{k}(\chi \circ \varphi)\left(\delta_{i}\right) D_{i}=\sum_{i=1}^{k} \Delta_{i} D_{i}, \\
d^{\prime} L_{\chi^{\prime}} & \equiv \sum_{i=1}^{k}\left(\chi^{\prime} \circ \varphi\right)\left(\delta_{i}\right) D_{i}=\sum_{i=1}^{k} \Delta_{i}^{\prime} D_{i},
\end{aligned}
$$


and

$$
M\left(L_{\chi+\chi^{\prime}}\right) \equiv \sum_{i=1}^{k}\left(\left(\chi+\chi^{\prime}\right) \circ \varphi\right)\left(\delta_{i}\right) D_{i} \equiv \sum_{i=1}^{k}\left(\left(\lambda \Delta_{i}+\lambda^{\prime} \Delta_{i}^{\prime}\right) \bmod (M)\right) \cdot D_{i}
$$

Since moreover $0<\lambda \Delta_{i}+\lambda^{\prime} \Delta_{i}^{\prime}<2 M$, we may write (identifying the divisor $L_{\chi}$ with the divisor $\left.\left(\sum_{i=1}^{k} \frac{\Delta_{i}}{d} D_{i}\right) \in \oplus_{i=1}^{k} \mathbb{Q} D_{i}\right)$

$$
L_{\chi}+L_{\chi^{\prime}}-L_{\chi+\chi^{\prime}}=\sum_{i=1}^{k} \epsilon_{D_{i}}^{\chi, \chi^{\prime}} D_{i}
$$

where $\epsilon_{D_{i}}^{\chi, \chi^{\prime}}:=1$ if $\lambda \Delta_{i}+\lambda^{\prime} \Delta_{i}^{\prime} \geq M$ and $\epsilon_{D_{i}}^{\chi, \chi^{\prime}}:=0$ otherwise.

The above equality is equivalent (as shown in [Par]) to the existence of the multiplication maps

$$
\mu_{\chi, \chi^{\prime}}: \mathcal{L}_{\chi}^{-1} \otimes \mathcal{L}_{\chi^{\prime}}^{-1} \rightarrow \mathcal{L}_{\chi+\chi^{\prime}}^{-1}
$$

which correspond to global sections of $\mathcal{L}_{\chi} \otimes \mathcal{L}_{\chi^{\prime}} \otimes \mathcal{L}_{\chi+\chi^{\prime}}^{-1}$ whose divisor is exactly equal to $\sum_{i=1}^{k} \epsilon_{D_{i}}^{\chi, \chi^{\prime}} D_{i}$.

Let in fact $\sigma_{i} \in \Gamma\left(X, \mathcal{O}\left(D_{i}\right)\right)$ be a section with $\operatorname{div}\left(\sigma_{i}\right)=D_{i}$ and set

$$
\epsilon_{\chi, \chi^{\prime}}^{i}:=\epsilon_{D_{i}}^{\chi, \chi^{\prime}}
$$

Then $\prod_{i} \sigma_{i}^{\epsilon^{i}}{ }^{\chi_{\chi^{\prime}}}$ is a global section of $\mathcal{L}_{\chi} \otimes \mathcal{L}_{\chi^{\prime}} \otimes \mathcal{L}_{\chi+\chi^{\prime}}^{-1}$ yielding the multiplication maps.

These sections define equations for the natural embedding

$$
i: X \hookrightarrow W:=\bigoplus_{\chi \in G^{*} \backslash\{1\}} \mathbb{V}\left(\mathcal{L}_{\chi}^{-1}\right)
$$

In fact, let $w_{\chi}$ be a fibre coordinate of $\mathbb{V}\left(\mathcal{L}_{\chi}^{-1}\right)$ : then $i(X)$ is defined by the equations

$$
w_{\chi} w_{\chi^{\prime}}=\prod_{\nu} \sigma_{\nu}^{\epsilon_{\chi, \chi^{\prime}}^{\nu}} w_{\chi+\chi^{\prime}}
$$

We infer the following

Corollary 1.9. If $Y$ and the $D_{i}$ 's are defined over a field $K$, then also $X$ is defined over $K$. 


\section{The construction}

We consider in $\mathbb{P}^{2}=\mathbb{P}_{\mathbb{C}}^{2}$ a complete quadrangle $\Delta$, i.e., the union of the six lines through four points $P_{0}, \ldots, P_{3}$ in general position.

Let $\pi: Y:=\hat{\mathbb{P}}^{2}\left(P_{0}, \ldots, P_{3}\right) \rightarrow \mathbb{P}^{2}$ be the Del Pezzo surface of degree 5 which is the blow up of the plane in the points $P_{0}, \ldots, P_{3}$. Denote by $E_{0}, \ldots, E_{3}$ the exceptional curves. Moreover, for $j=1,2,3$, let $L_{j}^{\prime}:=H-E_{0}-E_{j}$, where $H$ is the total transform in $Y$ of a line on $\mathbb{P}^{2}$, and let $L_{j}:=H-\sum_{i=1}^{3} E_{i}+E_{j}$. I.e., $L_{j}^{\prime}$ is the strict transform of the line in $\mathbb{P}^{2}$ through $P_{0}$ and $P_{j}$, whereas $L_{j}$ is the strict transform of the line in $\mathbb{P}^{2}$ through $E_{i}$ and $E_{k}$, where $\{i, j, k\}=\{1,2,3\}$.

The divisor $L_{1}+L_{2}+L_{3}+L_{1}^{\prime}+L_{2}^{\prime}+L_{3}^{\prime}+E_{0}+E_{1}+E_{2}+E_{3}$ on $Y$ has simple normal crossings and we shall denote it by $D$.

Remark 2.1. It is well known that $H^{2}(Y, \mathbb{Z})$ is freely generated by $H, E_{0}, \ldots, E_{3}$. Since

$$
H_{1}(Y-D, \mathbb{Z}) \cong \operatorname{coker}\left(r: H^{2 n-2}(Y) \rightarrow H^{2 n-2}(D) \cong \bigoplus_{i=1}^{k}\left[D_{i}\right] \mathbb{Z}\right),
$$

where $r$ is given by the intersection matrix

\begin{tabular}{|c|ccccc|}
\hline & $H$ & $E_{0}$ & $E_{1}$ & $E_{2}$ & $E_{3}$ \\
\hline$L_{1}^{\prime}$ & 1 & 1 & 1 & 0 & 0 \\
$L_{2}^{\prime}$ & 1 & 1 & 0 & 1 & 0 \\
$L_{3}^{\prime}$ & 1 & 1 & 0 & 0 & 1 \\
$L_{1}$ & 1 & 0 & 0 & 1 & 1 \\
$L_{2}$ & 1 & 0 & 1 & 0 & 1 \\
$L_{3}$ & 1 & 0 & 1 & 1 & 0 \\
$E_{0}$ & 0 & -1 & 0 & 0 & 0 \\
$E_{1}$ & 0 & 0 & -1 & 0 & 0 \\
$E_{2}$ & 0 & 0 & 0 & -1 & 0 \\
$E_{3}$ & 0 & 0 & 0 & 0 & -1 \\
\hline
\end{tabular}

we obtain

$$
H_{1}(Y-D, \mathbb{Z}) \cong\left(\bigoplus_{i=0}^{3} \mathbb{Z} e_{i} \oplus \bigoplus_{i=1}^{3} \mathbb{Z} l_{i} \oplus \bigoplus_{i=0}^{3} \mathbb{Z} l_{i}^{\prime}\right) / H^{2}(Y, \mathbb{Z}),
$$

where $e_{j}\left(\right.$ resp. $\left.l_{i}, l_{i}^{\prime}\right)$ is a (small) simple loop oriented counterclockwise around $E_{j}$ (resp. $L_{i}, L_{i}^{\prime}$ ). I.e., $H_{1}(Y-D, \mathbb{Z})$ has generators $e_{0}, \ldots, e_{3}, l_{1}, l_{2}, l_{3}, l_{1}^{\prime}, l_{2}^{\prime}, l_{3}^{\prime}$ and the relations are $e_{0}=l_{1}^{\prime}+l_{2}^{\prime}+l_{3}^{\prime}, e_{i}=l_{i}^{\prime}+l_{j}+l_{k}$ (here and in the following, $\{i, j, k\}=\{1,2,3\}), \sum l_{i}^{\prime}+\sum l_{i}=0$. In particular, $H_{1}(Y-D, \mathbb{Z})$ is free of rank 5 . 
We want to construct a smooth Galois cover $p: S \rightarrow Y$ with group $(\mathbb{Z} / 5 \mathbb{Z})^{2}$ branched exactly in $D$.

Such a Galois cover is determined by a surjective homomorphism $\varphi: \mathbb{Z}^{5} \cong$ $H_{1}(Y-D, \mathbb{Z}) \rightarrow(\mathbb{Z} / 5 \mathbb{Z})^{2}$ with certain conditions ensuring that $S$ is smooth and that the covering branches exactly in $D$.

We write

$$
\varphi\left(l_{i}\right)=:\left(\begin{array}{c}
x_{i} \\
y_{i}
\end{array}\right)=: u_{i}, \quad \varphi\left(l_{j}^{\prime}\right)=:\left(\begin{array}{c}
z_{j} \\
w_{j}
\end{array}\right)=: v_{j}, \quad \varphi\left(e_{h}\right)=: \epsilon_{h},
$$

where $x_{i}, y_{i}, z_{j}, w_{j} \in\{0, \ldots, 4\} \cong \mathbb{Z} / 5 \mathbb{Z}$.

Remark 2.2. It obviously follows from Remark 2.1 that each $\epsilon_{h}$ is determined by the $u_{i}$ 's, $v_{j}$ 's and that $\sum_{i} u_{i}+\sum_{j} v_{j}=0$.

In order to calculate the invariants (i.e., $p_{g}, K_{S}^{2}, q$ ) of the Galois covering given by the homomorphism $\varphi$, we have to calculate for each character $\chi \in\left((\mathbb{Z} / 5 \mathbb{Z})^{2}\right)^{*}$ the eigensheaf $\mathcal{L}_{\chi}$.

Before doing this let us work out first the two sets of conditions ensuring that our covering is

1) branched exactly in $D=L_{1}+L_{2}+L_{3}+L_{1}^{\prime}+L_{2}^{\prime}+L_{3}^{\prime}+E_{0}+E_{1}+E_{2}+E_{3}$; and that

2) $S$ is smooth.

Lemma 2.3. 1) If for all $i, u_{i}, v_{i}, \sum u_{h}, \epsilon_{i}=u_{i}+v_{j}+v_{k}$ are different from zero in $(\mathbb{Z} / 5 \mathbb{Z})^{2}$, then the covering $p: S \rightarrow Y$ is branched exactly in $L_{1}, L_{2}, L_{3}, L_{1}, L_{2}^{\prime}$, $L_{3}^{\prime}, E_{0}, \ldots, E_{3}$.

2) If the following pairs of vectors in $(\mathbb{Z} / 5 \mathbb{Z})^{2}$

$\left(u_{i}, v_{i}\right)$ for $i \in\{1,2,3\}$,

$\left.\begin{array}{l}\left(u_{1}, u_{1}+u_{2}+u_{3}\right),\left(u_{2}, u_{1}+u_{2}+u_{3}\right),\left(u_{3}, u_{1}+u_{2}+u_{3}\right), \\ \left(u_{1}, u_{1}+v_{2}+v_{3}\right),\left(u_{2}, u_{2}+v_{1}+v_{3}\right),\left(u_{3}, u_{3}+v_{1}+v_{2},\right. \\ \left(u_{1}+v_{2}+v_{3}, v_{i}\right),\end{array}\right\}$ for $i=2,3$,

$\left(u_{2}+v_{1}+v_{3}, v_{i}\right)$ for $i=1,3$,

$\left(u_{3}+v_{1}+v_{2}, v_{i}\right)$ for $i=1,2$

are linearly independent, then $S$ is smooth.

Proof. 1) is obvious.

2) follows from the fact that $S$ given by the homomorphism $\varphi$ is smooth if and only if the following condition holds: let $D_{1}, D_{2}$ be two non trivial irreducible subdivisors of the branch divisor of $p: S \rightarrow Y$ and let $d_{i}$ a small loop around $D_{i}$, then $\varphi\left(d_{1}\right)$ and $\varphi\left(d_{2}\right)$ are not in the same cyclic subgroup of $(\mathbb{Z} / 5 \mathbb{Z})^{2}$. 
Remark 2.4. Let $p: S \rightarrow Y$ be a $(\mathbb{Z} / 5 \mathbb{Z})^{2}$-Galois cover with $u_{i}$ and $v_{j}$ satisfying the two conditions of the above lemma. Then $S$ is a smooth minimal surface with $K_{S}^{2}=45$ and $\chi=5$. In fact, since the canonical divisor $K_{S}$ is the pull back of the rational divisor

$$
-3 H+\sum E_{i}+4 / 5 \sum E_{i}+4 / 5 \sum\left(L_{j}+L_{j}^{\prime}\right) \equiv 9 / 5 H-3 / 5 \sum E_{i}
$$

we get $K_{S}^{2}=\left(9^{2}-4 \cdot 3^{2}\right)=45$. Moreover, by the Noether's formula it suffices to compute that the Euler number $e(S)=15$. In fact, we get 25 times the Euler number of $\mathbb{P}^{2}$ blown up in 4 points, minus 20 times the Euler number of $10 \mathbb{P}^{1}$ 's plus 16 times the Euler number of 15 points: i.e., $25 \cdot 7-400+240=15$.

We are interested to find such surfaces with $q=0$, because then they will have geometric genus equal to 4 .

Given a character $\chi=(a, b) \in(\mathbb{Z} / 5 \mathbb{Z})^{2}$, let us determine $\mathcal{L}_{\chi}=\mathcal{L}_{(a, b)}$.

By the results of Section 1, we get

Proposition 2.5. Denoting by $[z]$ the residue class of $z$ modulo 5, we have the following formulae:

$$
\begin{aligned}
5 \mathcal{L}_{\chi} \equiv & \sum_{i=1}^{3} \chi\left(l_{i}\right) L_{i}+\sum_{i=1}^{3} \chi\left(l_{i}^{\prime}\right) L_{i}^{\prime}+\sum_{i=0}^{3} \chi\left(e_{i}\right) E_{i} \\
5 \mathcal{L}_{(a, b)} \equiv & \sum_{i=1}^{3}\left[a x_{i}+b y_{i}\right] L_{i}+\sum_{i=1}^{3}\left[a z_{i}+b w_{i}\right] L_{i}^{\prime}+\left[a\left(x_{1}+x_{2}+x_{3}\right)\right. \\
& \left.+b\left(y_{1}+y_{2}+y_{3}\right)\right] E_{0}+\sum_{i=1}^{3}\left[a\left(x_{i}+z_{j}+z_{k}\right)+b\left(y_{i}+w_{j}+w_{k}\right)\right] E_{i} .
\end{aligned}
$$

\section{The symmetries of the construction}

Definition 3.1. A six-tuple $\mathfrak{U}:=\left(u_{1}, u_{2}, u_{3}, v_{1}, v_{2}, v_{3}\right) \in\left((\mathbb{Z} / 5 \mathbb{Z})^{2} \backslash\{0\}\right)^{6}$ is said to be admissible if and only if

$$
u_{1}+u_{2}+u_{3}+v_{1}+v_{2}+v_{3}=0
$$

and moreover the two conditions of Lemma 2.3 are satisfied, i.e.:

a) for all $i, u_{i}, v_{i}, \sum u_{h}, \epsilon_{i}=u_{i}+v_{j}+v_{k}$ are different from zero in $(\mathbb{Z} / 5 \mathbb{Z})^{2}$;

b) the pairs of vectors listed in Lemma $2.3,2)$ are linearly independent in $(\mathbb{Z} / 5 \mathbb{Z})^{2}$. 
Remark 3.2. We have seen in the previous section that an admissible six-tuple $\mathfrak{U}$ induces a smooth Galois cover $p: S \rightarrow Y$ with Galois group $(\mathbb{Z} / 5 \mathbb{Z})^{2}$. Moreover, $S$ is a minimal surface of general type with $K_{S}^{2}=45$ and $\chi=5$. We recall that $S$ is a ball quotient, hence rigid.

Using MAGMA one sees that there are exactly 201600 admissible six-tuples. But of course a lot of them will lead to isomorphic surfaces. In order to understand how many non isomorphic surfaces (with $p_{g}=4$ ) we will get by this construction, we have to understand the symmetries.

Two admissible six-tuples $\mathfrak{U}, \mathfrak{U}^{\prime}$ obviously give isomorphic surfaces if there is an automorphism $\phi \in \mathrm{Gl}(2, \mathbb{Z} / 5 \mathbb{Z})$ such that $\phi(\mathfrak{U})=\mathfrak{U}^{\prime}$. On the other hand the group of biholomorphic automorphisms of $\mathbb{P}^{2} \backslash\left\{L_{1}, L_{2}, L_{3}, L_{1}^{\prime}, L_{2}^{\prime}, L_{3}^{\prime}\right\}$ equals $\mathfrak{S}_{5}$ (cf. [Ter]). The action of $\mathfrak{S}_{5}$ on the set of admissible six tuples is generated by the following transformations:

(01): $\left(u_{1}, u_{2}, u_{3}, v_{1}, v_{2}, v_{3}\right) \rightarrow\left(u_{1}, u_{3}+v_{1}+v_{2}, u_{2}+v_{1}+v_{3}, u_{1}+u_{2}+u_{3}, v_{2}, v_{3}\right)$;

$(02):\left(u_{1}, u_{2}, u_{3}, v_{1}, v_{2}, v_{3}\right) \rightarrow\left(u_{3}+v_{1}+v_{2}, u_{2}, u_{1}+v_{2}+v_{3}, v_{1}, u_{1}+u_{2}+u_{3}, v_{3}\right)$;

$(03):\left(u_{1}, u_{2}, u_{3}, v_{1}, v_{2}, v_{3}\right) \rightarrow\left(u_{2}+v_{1}+v_{3}, u_{3}+v_{1}+v_{2}, u_{3}, v_{1}, v_{2}, u_{1}+u_{2}+u_{3}\right)$;

$(04):\left(u_{1}, u_{2}, u_{3}, v_{1}, v_{2}, v_{3}\right) \rightarrow\left(u_{1}, u_{2}, u_{3}, u_{1}+v_{2}+v_{3}, u_{2}+v_{1}+v_{3}, u_{3}+v_{1}+v_{2}\right)$.

It is easy to see that these four transpositions generate the action of a group isomorphic to $\mathfrak{S}_{5}$.

We consider now the group $g$ acting on the set of admissible six-tuples 8 , which is generated by $\mathfrak{S}_{5}$ and $\mathrm{Gl}(2, \mathbb{Z} / 5 \mathbb{Z})$. Then $g$ is a quotient of $\mathrm{Gl}(2, \mathbb{Z} / 5 \mathbb{Z}) \times \mathfrak{S}_{5}$ (the actions commute, being given by multiplication on the right, respectively on the left). A MAGMA computation, reproduced in the appendix, reveals that $g$ has four orbits on $\&$ (indeed, the simple minded idea is to calculate the cardinality of these 4 orbits, and see that their sum equals the cardinality of the set of admissible six-tuples).

Representatives for these orbits are

$$
\begin{aligned}
& \mathfrak{U}_{1}=\left(\left(\begin{array}{l}
1 \\
0
\end{array}\right),\left(\begin{array}{l}
1 \\
0
\end{array}\right),\left(\begin{array}{l}
0 \\
1
\end{array}\right),\left(\begin{array}{l}
2 \\
1
\end{array}\right),\left(\begin{array}{l}
2 \\
1
\end{array}\right),\left(\begin{array}{l}
4 \\
2
\end{array}\right)\right) ; \\
& \mathfrak{U}_{2}=\left(\left(\begin{array}{l}
1 \\
0
\end{array}\right),\left(\begin{array}{l}
1 \\
0
\end{array}\right),\left(\begin{array}{l}
0 \\
1
\end{array}\right),\left(\begin{array}{l}
2 \\
1
\end{array}\right),\left(\begin{array}{l}
4 \\
2
\end{array}\right),\left(\begin{array}{l}
2 \\
1
\end{array}\right)\right) ; \\
& \mathfrak{U}_{3}=\left(\left(\begin{array}{l}
1 \\
0
\end{array}\right),\left(\begin{array}{l}
1 \\
0
\end{array}\right),\left(\begin{array}{l}
0 \\
1
\end{array}\right),\left(\begin{array}{l}
4 \\
1
\end{array}\right),\left(\begin{array}{l}
3 \\
2
\end{array}\right),\left(\begin{array}{l}
1 \\
1
\end{array}\right)\right) ; \\
& \mathfrak{U}_{4}=\left(\left(\begin{array}{l}
1 \\
0
\end{array}\right),\left(\begin{array}{l}
1 \\
0
\end{array}\right),\left(\begin{array}{l}
0 \\
1
\end{array}\right),\left(\begin{array}{l}
1 \\
1
\end{array}\right),\left(\begin{array}{l}
0 \\
3
\end{array}\right),\left(\begin{array}{l}
2 \\
0
\end{array}\right)\right) .
\end{aligned}
$$


The orbit of $\mathfrak{U}_{1}$ has length 28800 , whereas the orbits of $\mathfrak{U}_{2}, \mathfrak{U}_{3}, \mathfrak{U}_{4}$ have respective length 57600 .

In particular we see that $g \cong \mathrm{Gl}(2, \mathbb{Z} / 5 \mathbb{Z}) \times \mathfrak{S}_{5}$.

We have moreover:

Theorem 3.3. Let $S_{i}$ be the minimal smooth surface of general type with $K^{2}=45$ and $\chi=5$ obtained from the covering induced by the admissible six-tuple $\mathfrak{U}_{i}$, where $i \in\{1,2,3,4\}$. Then we have that $S_{3}$ is regular (i.e., $q\left(S_{3}\right)=0$ ), whereas $q\left(S_{i}\right)=2$ for $i \neq 3$.

In particular, $S_{3}$ is the unique minimal surface with $K_{S}^{2}=45$ and $p_{g}=4$ obtained as a $(\mathbb{Z} / 5 \mathbb{Z})^{2}$-cover of $\mathbb{P}^{2}$ branched exactly in a complete quadrangle of the complex projective plane.

Proof. We will calculate the geometric genus of $S=S_{3}$, using the formula

$$
H^{0}\left(S, \mathcal{O}_{S}\left(K_{S}\right)\right)=\bigoplus_{(a, b) \in G^{*}} H^{0}\left(Y, \mathcal{O}_{Y}\left(K_{Y}\right) \otimes \mathcal{L}_{(a, b)}\right)
$$

Applying Proposition 2.5 we obtain the following table for the divisors $L_{a, b}$ yielding the character sheaves $\mathcal{L}_{(a, b)}=\mathcal{O}_{Y}\left(L_{a, b}\right)$ :

\begin{tabular}{|c|c|c|c|c|c|}
\hline $\mathcal{L}_{(a, b)}$ & $a=0$ & $a=1$ & $a=2$ & $a=3$ & $a=4$ \\
\hline$b=0$ & 0 & $\begin{array}{c}2 H-E_{1}-E_{2} \\
-E_{3}\end{array}$ & $2 H-E_{1}-E_{2}$ & $\begin{array}{c}3 H-E_{0}-2 E_{1} \\
-E_{2}-E_{3}\end{array}$ & $\begin{array}{c}3 H-E_{0}-2 E_{1} \\
-E_{2}\end{array}$ \\
\hline$b=1$ & $H$ & $H$ & $3 H-E_{0}-E_{1}$ & $3 H-E_{0}-E_{1}$ & $3 H-E_{0}-E_{1}$ \\
& & $-E_{2}-E_{3}$ & $-2 E_{2}-E_{3}$ & $-E_{2}-E_{3}$ \\
\hline$b=2$ & $2 H-E_{1}-E_{3}$ & $2 H-E_{1}-E_{2}$ & $2 H-E_{0}-E_{1}$ & $3 H-E_{0}-E_{1}$ & $3 H-2 E_{0}-E_{1}$ \\
& & $-E_{3}$ & $-E_{2}$ & $-E_{2}-E_{3}$ & $-E_{2}-E_{3}$ \\
\hline$b=3$ & $2 H-E_{2}-E_{3}$ & $3 H-E_{0}-E_{1}$ & $2 H-E_{0}-E_{3}$ & $2 H-E_{0}$ & $4 H-2 E_{0}-E_{1}$ \\
& & $-E_{2}-E_{3}$ & & & $-2 E_{2}-2 E_{3}$ \\
\hline$b=4$ & $3 H-E_{1}-E_{2}$ & $2 H-E_{0}-E_{3}$ & $3 H-E_{0}-E_{1}$ & $3 H-2 E_{0}-E_{1}$ & $3 H-2 E_{0}-E_{1}$ \\
& $-2 E_{3}$ & & $-E_{2}-2 E_{3}$ & $-E_{2}-E_{3}$ & $-E_{2}$ \\
\hline
\end{tabular}

We see immediately that $H^{0}\left(Y, \mathcal{O}_{Y}\left(K_{Y}\right) \otimes \mathcal{L}_{(a, b)}\right)=0$ for all $(a, b) \notin\{(2,1)$, $(3,2),(1,3),(4,1)\}$ and $H^{0}\left(Y, \mathcal{O}_{Y}\left(K_{Y}\right) \otimes \mathcal{L}_{(a, b)}\right) \cong \mathbb{C}$ for $(a, b) \in\{(2,1),(3,2)$, $(1,3),(4,1)\}$, i.e., $p_{g}\left(S_{3}\right)=4$. This proves the claim for $S_{3}$.

The geometric genus of the remaining surfaces is calculated in exactly the same way. 
Vol. 83 (2008)

\section{The canonical map}

In the previous section we have constructed a minimal surface $S$ of general type with $K_{S}^{2}=45, p_{g}=4$ and $q(S)=0$. We want now to understand the behaviour of the canonical map of $S$.

For $(a, b) \in(\mathbb{Z} / 5)^{2}$ we write

$$
\begin{gathered}
\delta_{i}(a, b):=\left[a x_{i}+b y_{i}\right], \\
\lambda_{j}(a, b):=\left[a z_{j}+b w_{j}\right], \\
\mu_{0}(a, b):=\left[a\left(x_{1}+x_{2}+x_{3}\right)+b\left(y_{1}+y_{2}+y_{3}\right)\right], \\
\mu_{h}(a, b):=\left[a\left(x_{h}+z_{j}+z_{k}\right)+b\left(y_{h}+w_{j}+w_{k}\right)\right] .
\end{gathered}
$$

Then we know that

$$
5 \mathcal{L}_{(a, b)}=\sum_{i=1}^{3} \delta_{i}(a, b) L_{i}^{\prime}+\sum_{j=1}^{3} \lambda_{j}(a, b) L_{j}+\sum_{h=0}^{3} \mu_{h}(a, b) E_{h} .
$$

Denote by $R_{1}, \ldots, R_{10}$ the ramification divisors of $p: S \rightarrow Y$ lying over $L_{1}^{\prime}, L_{2}^{\prime}$, $L_{3}^{\prime}, L_{1}, L_{2}, L_{3}, E_{0}, \ldots, E_{3}$ : it is easy to see that they are all irreducible genus 2 curves. Further, let $x_{i}$ be a local equation of $R_{i}$. We already saw that $H^{0}\left(S, \mathcal{O}_{S}\left(K_{S}\right)\right)$ is the direct sum of 4 one dimensional eigenspaces $H^{0}\left(S, \mathcal{O}_{S}\left(K_{S}\right)\right)_{(a, b)} \cong H^{0}\left(\left(Y, \mathcal{O}_{Y}\left(K_{Y}\right) \otimes\right.\right.$ $\left.\mathcal{L}_{(a, b)}\right) \cong H^{0}\left(\mathbb{P}^{2}, \mathcal{O}_{\mathbb{P}^{2}}\right)$. Then a basis of $H^{0}\left(S, \mathcal{O}_{S}\left(K_{S}\right)\right)$ is given by

$$
\begin{aligned}
\left\{x_{1}^{4-\delta_{1}(a, b)}\right. & \cdot x_{2}^{4-\delta_{2}(a, b)} \cdot x_{3}^{4-\delta_{3}(a, b)} \cdot x_{4}^{4-\lambda_{1}(a, b)} \cdot x_{5}^{4-\lambda_{2}(a, b)} \cdot x_{6}^{4-\lambda_{3}(a, b)} \\
& \left.\cdot x_{7}^{4-\mu_{0}(a, b)} \ldots x_{10}^{4-\mu_{3}(a, b)} \mid H^{0}\left(Y, \mathcal{O}_{Y}\left(K_{Y}\right) \otimes \mathcal{L}_{(a, b)}\right) \neq 0\right\} .
\end{aligned}
$$

It is easy to compute the table giving the numbers $\lambda_{i}, \delta_{j}$ and $\mu_{h}$ for $(a, b) \in$ $\{(2,1),(3,2),(1,3),(4,1)\}$ :

\begin{tabular}{|c|c|c|c|c|c|c|c|c|c|c|}
\hline$(a, b)$ & $\delta_{1}$ & $\delta_{2}$ & $\delta_{3}$ & $\lambda_{1}$ & $\lambda_{2}$ & $\lambda_{3}$ & $\mu_{0}$ & $\mu_{1}$ & $\mu_{2}$ & $\mu_{3}$ \\
\hline$(1,3)$ & 1 & 1 & 3 & 2 & 4 & 4 & 0 & 4 & 2 & 4 \\
\hline$(2,1)$ & 2 & 2 & 1 & 4 & 3 & 3 & 0 & 3 & 4 & 3 \\
\hline$(3,2)$ & 3 & 3 & 2 & 4 & 3 & 0 & 3 & 1 & 2 & 4 \\
\hline$(4,1)$ & 4 & 4 & 1 & 2 & 4 & 0 & 4 & 3 & 1 & 2 \\
\hline
\end{tabular}

Therefore we have the following result.

Lemma 4.1. A basis for $H^{0}\left(S, \mathcal{O}_{S}\left(K_{S}\right)\right)$ is given by $\left\{x_{1}^{3} x_{2}^{3} x_{3} x_{4}^{2} x_{7}^{4} x_{9}^{2}, x_{1}^{2} x_{2}^{2} x_{3}^{3} x_{5} x_{6} x_{7}^{4} x_{8} x_{10}, x_{1} x_{2} x_{3}^{2} x_{5} x_{6}^{4} x_{7} x_{8}^{3} x_{9}^{2}, x_{3}^{3} x_{4}^{2} x_{6}^{4} x_{8} x_{9}^{3} x_{10}^{2}\right\}$. 
We can now prove the following

Theorem 4.2. 1) The canonical map $\phi_{K}$ of $S$ has $R_{3}$ as fixed part and its movable part has five base points. We have $R_{3}^{2}=-1$ and $K_{S} \cdot R_{3}=3$. The base points of $K_{S}-R_{3}$ are $x_{1} \cap x_{4}$ (of type $(1,1)$ ), $x_{1} \cap x_{8}$ (of type $(1,1,1)$ ), $x_{2} \cap x_{9}$ (of type $(2,1,1)), x_{3} \cap x_{7}$ (of type $(2,1,1)$ ), $x_{6} \cap x_{9}$ (of type $(1,1)$ ).

2) The canonical map is birational and its image in $\mathbb{P}^{3}$ has degree 19.

In order to keep the formulation of the above theorem as simple as possible we adopted the notation: a base point $p$ of $K-R_{3}$ on $S$ is of type $\left(n_{1}, n_{2}, \ldots, n_{k}\right)$ iff $p$ is a $n_{1}$-tuple base point of $K-R_{3}$, after one blow up the strict transform of $K-R_{3}$ has a $n_{2}$-tuple base point and so on.

Proof. It is immediate from the description of the basis of $H^{0}\left(S, \mathcal{O}_{S}\left(K_{S}\right)\right)$ given in Lemma 4.1 that $R_{3}$ is exactly the fixed part of $\left|K_{S}\right|$. It is easy to see that the base points of $\left|K-R_{3}\right|$ are exactly $x_{1} \cap x_{4}, x_{1} \cap x_{8}, x_{2} \cap x_{9}, x_{3} \cap x_{7}, x_{6} \cap x_{9}$. Next we will see which kind of base points we have and whether there are still infinitely near base points.

1) $x_{1} \cap x_{4}$ : locally around this point $\left|K-R_{3}\right|$ is given by $x_{1}^{3} x_{4}^{2}, x_{1}^{2}, x_{1}, x_{4}^{2}$. The ideal generated is the ideal $\left(x_{1}, x_{4}^{2}\right)$, thus $K-R_{3}$ has a base point of type $(1,1)$ in $x_{1} \cap x_{4}$.

2) $x_{1} \cap x_{8}$ : locally around this point $\left|K-R_{3}\right|$ is given by $x_{1}^{3}, x_{1}^{2} x_{8}, x_{1} x_{8}^{3}, x_{8}$. The ideal generated is the ideal $\left(x_{1}^{3}, x_{8}\right)$, thus $K-R_{3}$ has a base point of type $(1,1,1)$ in $x_{1} \cap x_{8}$.

Similarly in the three remaining cases we see that:

3) $\left|K-R_{3}\right|$ has a base point of type $(2,1,1)$ in $x_{2} \cap x_{9}$ (ideal $\left(x_{2}^{2}, x_{2} x_{9}^{2}\right)$ ).

4) $\left|K-R_{3}\right|$ has a base point of type $(2,1,1)$ in $x_{3} \cap x_{7}$ (ideal $\left(x_{3}^{2}, x_{7}^{4}, x_{3} x_{7}\right)$ ).

5) $\left|K-R_{3}\right|$ has a base point of type $(1,1)$ in $x_{6} \cap x_{9}$ (ideal $\left(x_{6}, x_{9}^{2}\right)$ ).

Therefore we get $\operatorname{deg} \phi_{K} \operatorname{deg} \phi_{K}(S)=\left(K-R_{3}\right)^{2}-2 \cdot 4-11=19$. Here we use that $R_{3}$ has self intersection -1 and genus 2 . It follows immediately that $\operatorname{deg} \phi_{K}=1$ and that the canonical image has degree 19.

The following is an answer to a question by Junho Lee.

Corollary 4.3. There exist surfaces of general type $S$ with birational canonical map such that the canonical system of each deformation of $S$ has base points.

There remains finally the statement of the main theorem concerning the first homology group of the four ball quotients which are obtained as $(\mathbb{Z} / 5)^{2}$-Galois covers of $\mathbb{P}^{2}$ branched on a complete quadrangle $\Delta$. This will be done in the next section. 


\section{Fundamental group and first homology group of $S$}

Let $S$ be a surface as above with $K^{2}=45$ and $\chi=5$ constructed as $(\mathbb{Z} / 5 \mathbb{Z})^{2}$ cover of the plane ramified exactly over a complete quadrangle. The first aim of this section is to find a description of the fundamental group of $S$.

Recall that the covering $p: S \rightarrow Y=\hat{\mathbb{P}}^{2}\left(P_{0}, \ldots, P_{3}\right)$ is given by the homomorphism $\varphi: \pi_{1}\left(\mathbb{P}^{2}-\Delta\right) \rightarrow H^{1}\left(\mathbb{P}^{2}-\Delta, \mathbb{Z} / 5 \mathbb{Z}\right) \rightarrow(\mathbb{Z} / 5 \mathbb{Z})^{2}$. I.e., we have the exact sequence

$$
1 \rightarrow \pi_{1}(S-R) \cong \operatorname{ker}(\varphi) \rightarrow \pi_{1}\left(\mathbb{P}^{2}-\Delta\right) \rightarrow(\mathbb{Z} / 5 \mathbb{Z})^{2} \rightarrow 0,
$$

where $R \subset S$ is the ramification divisor of $p: S \rightarrow Y$, i.e., $R=\sum_{i=1}^{10} R_{i}$. Let $\alpha_{i}$ be a small geometric loop around $R_{i}$, for each $i \in\{1, \ldots, 10\}$, and denote by $\left\langle\alpha_{1}, \ldots, \alpha_{10}\right\rangle$ the normal subgroup of $\pi_{1}(S-R)$ generated by $\alpha_{1}, \ldots, \alpha_{10}$.

Then $\pi_{1}(S)=\pi_{1}(S-R) /\left\langle\alpha_{1}, \ldots, \alpha_{10}\right\rangle$.

We first have to give a suitable presentation of $\pi_{1}\left(\mathbb{P}^{2}-\Delta\right)$. For this we use a result of T. Terada ([Ter]). We change the notation and use Terada's notation (cf. Figure 1).

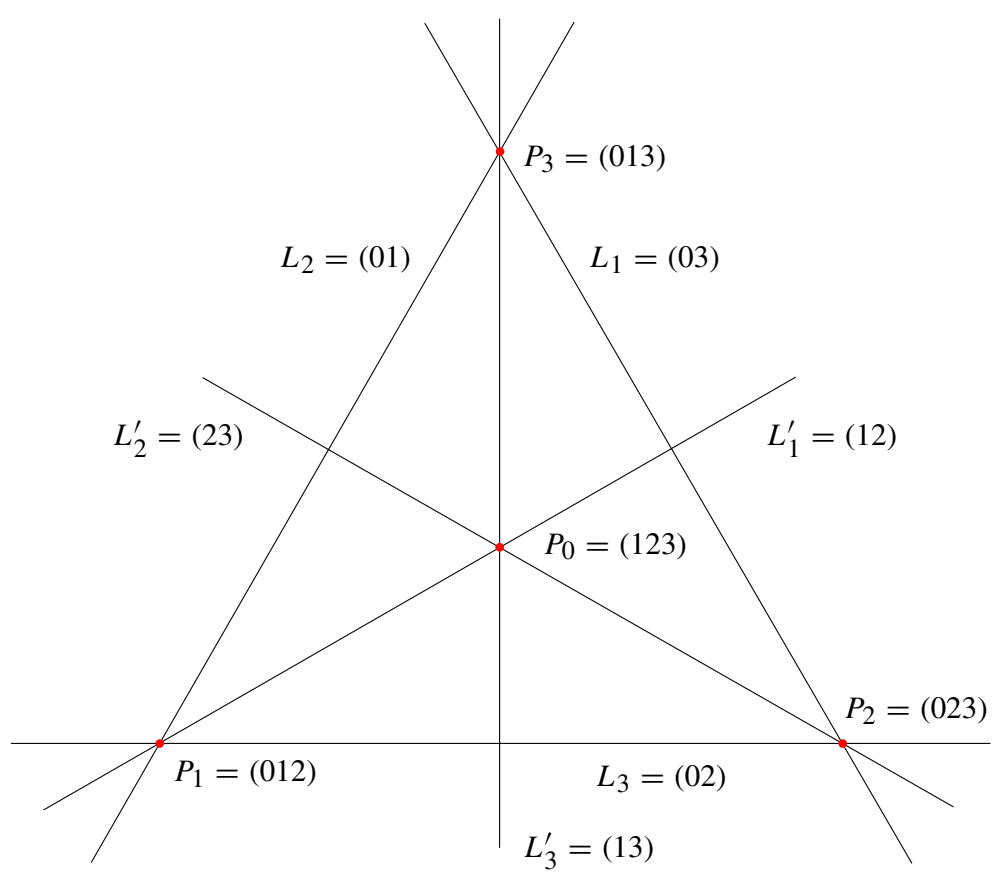

Figure 1. A complete quadrangle in $\mathbb{P}^{2}$. 
Let $a \in \mathbb{P}^{2}-\Delta$ and denote by $\rho(i j)$ resp. $\rho(i j k), 0 \leq i<j<k \leq 3$ a small loop starting from $a$ around $(i j)$ resp. around the exceptional divisor of the blow up in $(i j k)$ defined as in [Ter]. Then Terada shows the following result.

Theorem 5.1 (Terada). $\pi_{1}\left(\mathbb{P}^{2}-\Delta, a\right)$ is generated by $\rho(i j), i<j$ and the relations are

$$
\begin{aligned}
{[\rho(i j) \rho(i k) \rho(j k), \rho(i j)] } & =1, \\
{[\rho(i j) \rho(i k) \rho(j k), \rho(i k)] } & =1, \\
{[\rho(i j) \rho(i k) \rho(j k), \rho(j k)] } & =1, \quad \text { for all } i<j<k ; \\
\rho(01) \rho(02) \rho(12) \rho(03) \rho(13) \rho(23) & =1 .
\end{aligned}
$$

On the other hand, it is well known that $\pi_{1}\left(\mathbb{P}^{2}-\Delta\right)$ is isomorphic to the quotient of the pure braid group of four points by its center $\mathcal{P}_{4} / Z_{4}$. Let $\sigma_{i}$ be the standard generators of Artin's braid group. Then we have

$$
\rho(i j)=\sigma_{i}^{-1} \sigma_{i+1}^{-1} \ldots \sigma_{j-2}^{-1} \sigma_{j-1}^{2} \sigma_{j-2} \ldots \sigma_{i} .
$$

We will show that $\rho(i j) \rho(i k) \rho(j k)$ generates the center of $\mathcal{P}_{3} \cong \pi_{1}\left(\mathbb{C}^{2}-Z\right)$, where $Z$ is the union of three lines through one point. This implies, in view of the geometric exact sequence

$$
1 \rightarrow Z_{3} \rightarrow \mathcal{P}_{3} \rightarrow \pi_{1}\left(\mathbb{P}^{1} \backslash\{0,1, \infty\}\right) \rightarrow 1,
$$

that $\rho(i j) \rho(i k) \rho(j k)$ represents a simple loop around the exceptional divisor over $(i j k)$ in $\pi_{1}\left(\mathbb{P}^{2}-\Delta\right)$.

We have in fact that $\rho(01) \rho(02) \rho(12)=\sigma_{0}^{2} \sigma_{0}^{-1} \sigma_{1}^{2} \sigma_{0} \sigma_{1}^{2}$, is the standard generator of the centre of $\mathcal{P}_{3}$ according to Chow's theorem (see Corollary 1.8.4, p. 28 of [Bir]).

The remaining three cases are treated in the same way.

We summarize our result in the following

Proposition 5.2. Let $\mathfrak{U}:=\left(u_{1}, u_{2}, u_{3}, v_{1}, v_{2}, v_{3}\right)$ be an admissible six-tuple and let $S$ be the smooth surface constructed from $\mathfrak{U}$.

Let $\Phi: \pi_{1}\left(\mathbb{P}^{2}-\Delta\right) \rightarrow(\mathbb{Z} / 5)^{2}$ be the morphism given by $\rho(01) \mapsto v_{2}, \rho(02) \mapsto$ $v_{3}, \rho(12) \mapsto u_{1}, \rho(03) \mapsto v_{1}, \rho(13) \mapsto u_{3}, \rho(23) \mapsto u_{2}$.

Then $\pi_{1}(S) \cong \operatorname{ker} \Phi / N$, where $N$ is the subgroup of $\operatorname{ker} \Phi$ normally generated by

$$
\begin{aligned}
& \rho(01)^{5}, \quad \rho(02)^{5}, \quad \rho(12)^{5}, \quad \rho(02)^{5}, \quad \rho(13)^{5}, \quad \rho(23)^{5}, \quad(\rho(01) \rho(02) \rho(12))^{5}, \\
& (\rho(02) \rho(03) \rho(23))^{5}, \quad(\rho(01) \rho(03) \rho(13))^{5}, \quad(\rho(12) \rho(13) \rho(23))^{5} \text {. }
\end{aligned}
$$

From the above description of the fundamental group of $S$, using the method of Reidemeister-Schreier, its abelianization $H_{1}(S, \mathbb{Z})$ can be calculated via a MAGMA program which is reproduced in the appendix. 


\section{Appendix}

The following program calculates the admissible six-tuples.

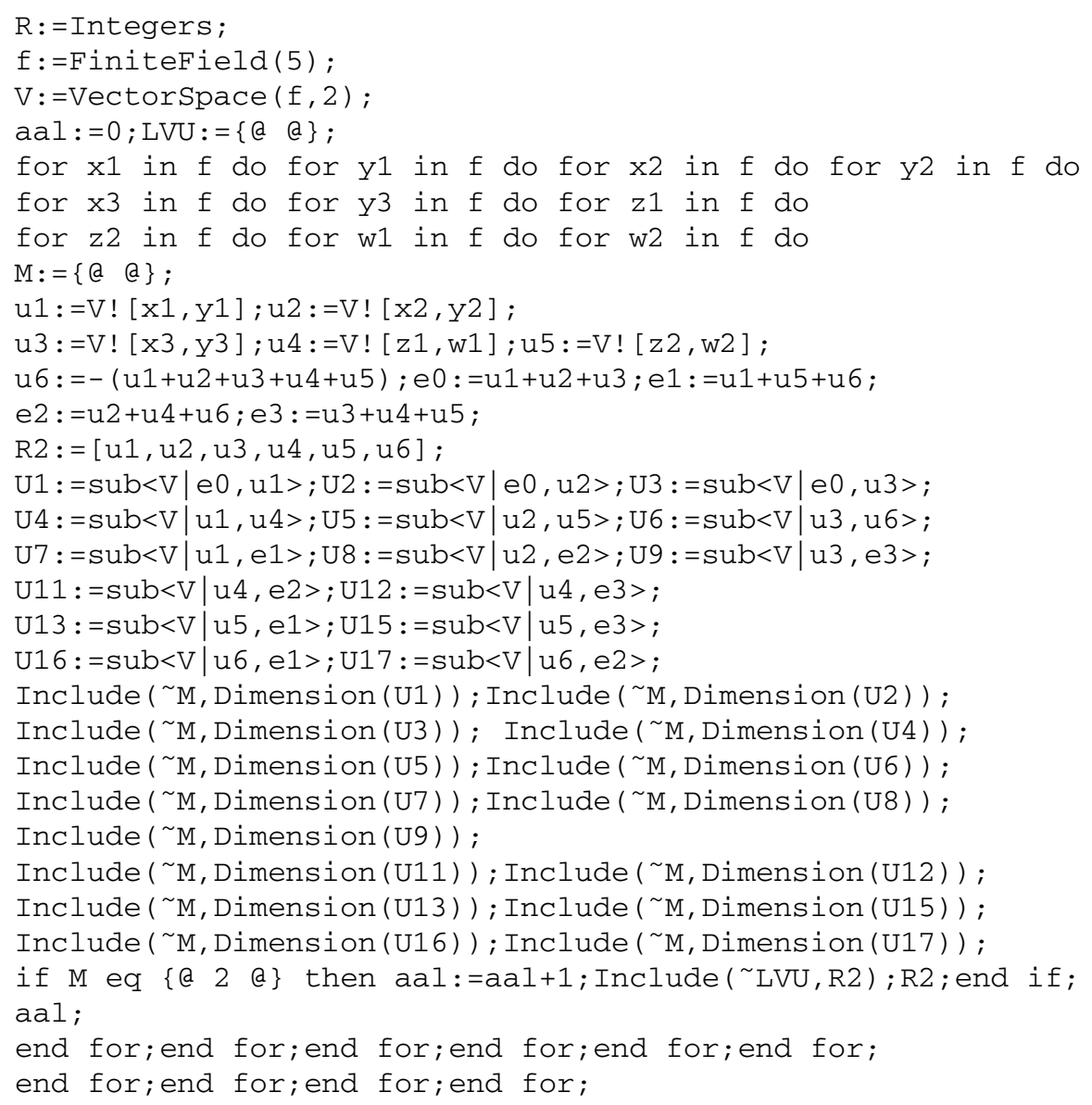

The following script calculates the length of the orbits of four admissible sixtuples. We skip the verification that these are four distinct orbits.

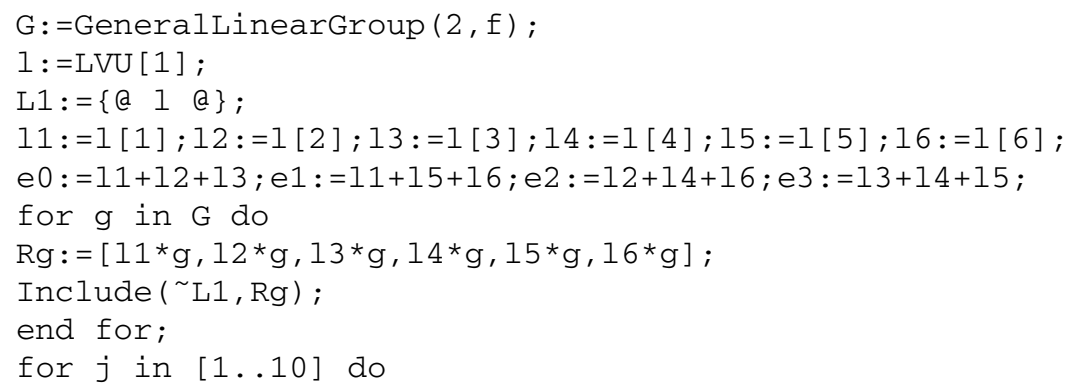




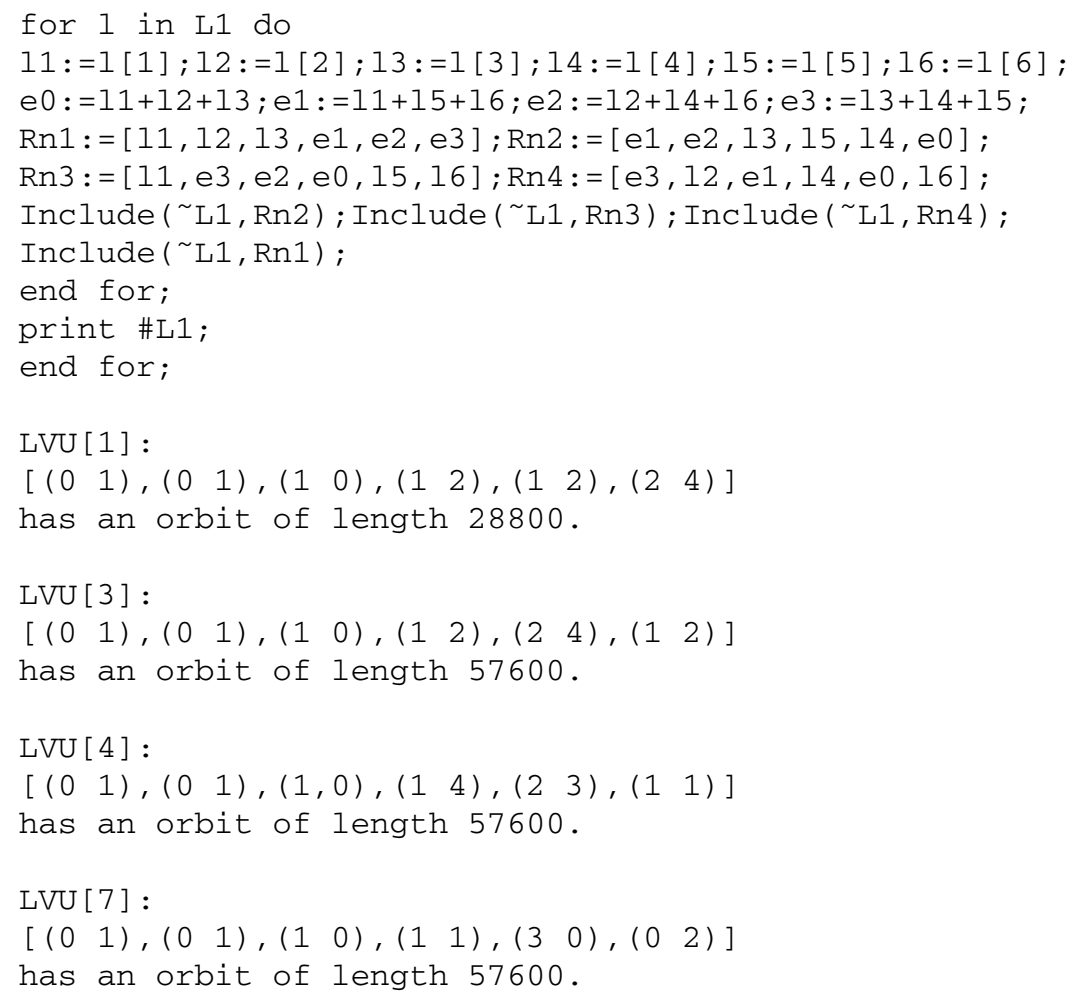

The following script calculates the abelianization of the fundamental group of $S_{3}$.

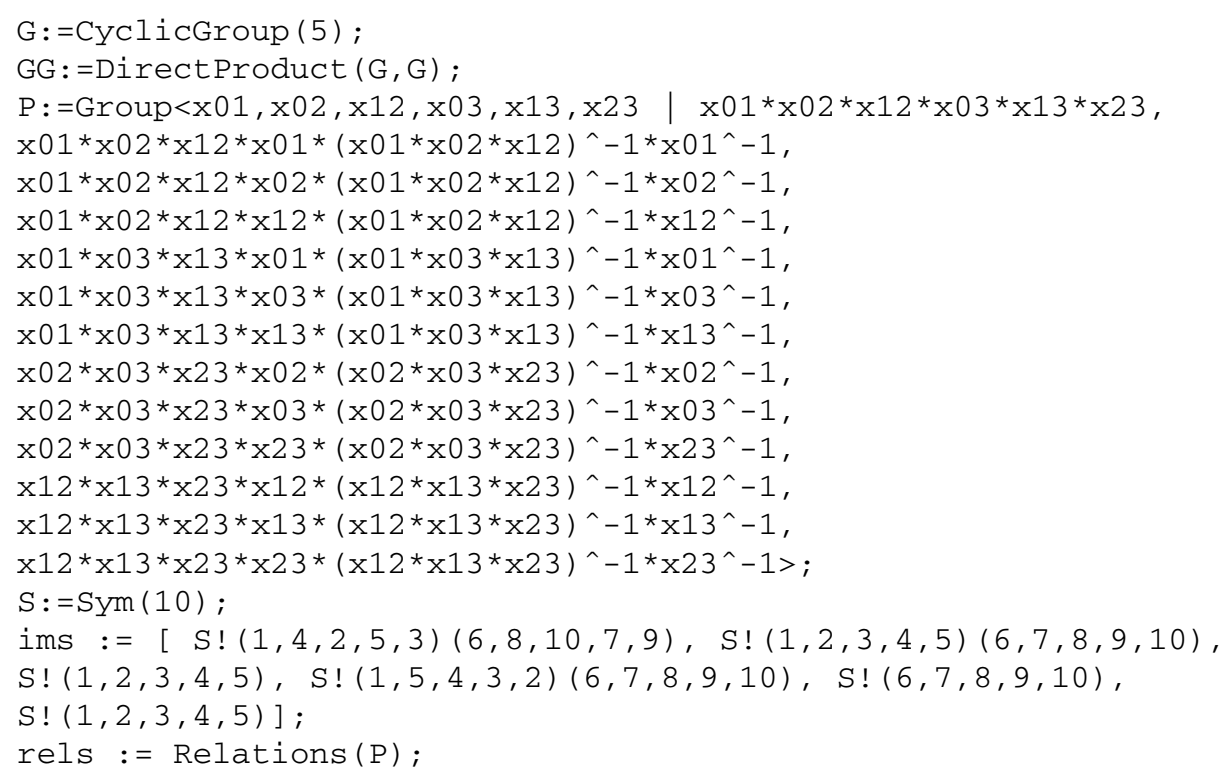




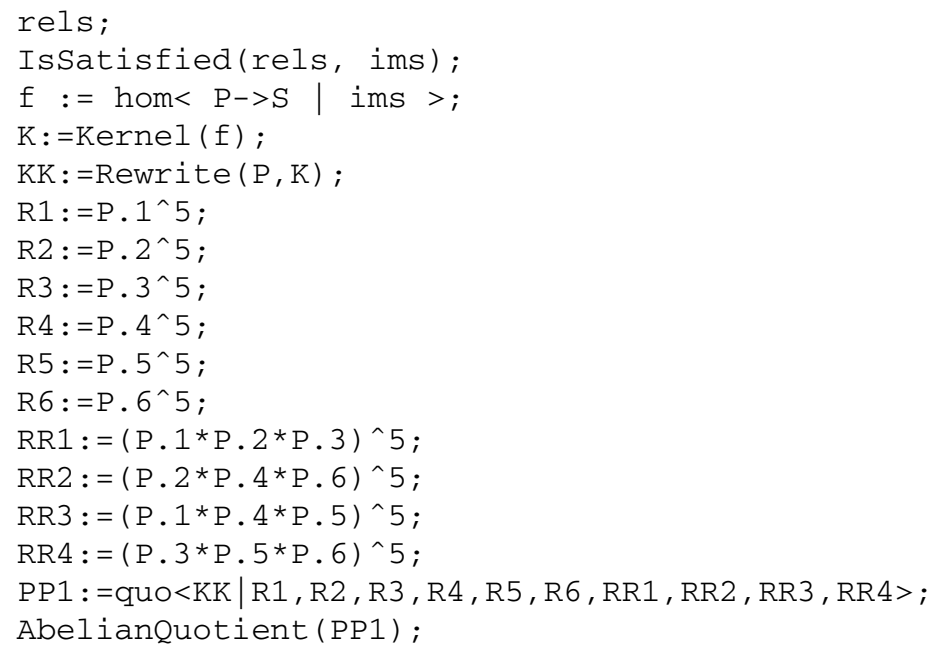

Acknowledgement. We are grateful to Fritz Grunewald for his invaluable help in the explicit calculations leading to Theorem 3.3.

This research was performed in the realm of the DFG Schwerpunkt "Globale methoden in der komplexen Geometrie".

\section{References}

[BPV] W. Barth, C. Peters, A. Van de Ven, Compact complex surfaces. Ergeb. Math. Grenzgeb. (3) 4, Springer-Verlag, Berlin 1984. Zbl 0718.14023 MR 0749574

$[\mathrm{BHH}]$ G. Barthel, F. Hirzebruch, T. Höfer, Geradenkonfigurationen und Algebraische Flächen. Aspekte der Math. D4, Vieweg, Braunschweig 1987. Zbl 0645.14016 MR 0912097

[Ba-Ca] I. Bauer, F. Catanese, Symmetry and Variation of Hodge Structures. Asian J. Math. 8 (2) (2004), 363-390. Zbl 1070.14013 MR 2129542

[Bea] A. Beauville, Surfaces algébriques complexes. Astérisque 54 (1978). Zbl 0394.14014 MR 0485887

[Bir] J. Birman, Braids, links and mapping class groups. Ann. of Math. Stud. 82, Princeton University Press, Princeton, N. J., 1975. Zbl 0305.57013 MR 0375281

[Bur] P. Burniat, Modèles de surfaces canoniques normales de $S_{3}$ et de genre linéaire $11 \leq$ $p^{(1)} \leq 17$. In Deuxième Colloque de Géométrie Algébrique (Liège, 1952), Georges Thone, Liège; Masson \& Cie, Paris 1952, 185-210. Zbl 0047.39901 MR 0054285

[Cat1] F. Catanese, On the moduli space of surfaces of general type. J. Differential Geom. 19 (1984), 483-515. Zbl 0549.14012 MR 0755236

[Cat2] F. Catanese, Singular bidouble covers and the construction of interesting algebraic surfaces. In Algebraic geometry: Hirzebruch 70, Contemp. Math. 241, Amer. Math. Soc., Providence, RI, 1999, 97-120. Zbl 0964.14012 MR 1718139 
[Cil] C. Ciliberto,Canonical surfaces with $p_{g}=p_{a}=4$ and $K^{2}=5, \ldots, 10$. Duke Math. J. 48 (1) (1981), 121-157. Zbl 0468.14011 MR 0610180

[Enr] F. Enriques, Le Superficie Algebriche. Zanichelli, Bologna 1949. Zbl 0036.37102 MR 0031770

[Fran] A. Franchetta, Su alcuni esempi di superficie canoniche. Rend. Sem. Mat. Roma 3 (1939), 23-28. JFM 65.0722.04 Zbl 0021.15202

[Hir] F. Hirzebruch, Arrangements of lines and algebraic surfaces. In Arithmetic and geometry, Vol. II, Progr. Math 36, Birkhäuser, Boston, Mass., 1983, 113-140. Zbl 0527.14033 MR 0717609

[Ish1] M.-N. Ishida, The irregularities of Hirzebruch's examples of surfaces of general type with $c_{1}^{2}=3 c_{2}$. Math. Ann. 262 (1983), 407-420. Zbl 0488.14009 MR 0692865

[Ish2] M.-N. Ishida, Hirzebruch's examples of surfaces of general type with $c_{1}^{2}=3 c_{2}$. In Algebraic Geometry, Lecture Notes in Math. 1016, Springer-Verlag, Berlin 1983, 412-431. Zbl 0558.14026 MR 0726436

[Kod] K. Kodaira, On characteristic systems of families of surfaces with ordinary singularities in a projective space. Amer. J. Math. 87 (1965), 227-256. Zbl 0145.43802 MR 0176380

[Lie] C. Liedtke, Singular abelian covers of algebraic surfaces. Manuscripta Math. 112 (2003), 375-390. Zbl 1044.14016 MR 2067044

[Max] E. A. Maxwell, Regular canonical surfaces of genus three and four. Proc. Cambridge Philos. Soc. 23 (1937), 306-310. Zbl 0017.03103

[Miy] Y. Miyaoka, Algebraic surfaces with positive indices. In Classification of algebraic and analytic manifolds. (Katata, 1982), Progr. Math. 39, Birkhäuser, Boston, Mass., 281-301. Zbl 0584.14022 MR 0728611

[Mum] D. Mumford, An algebraic surface with $K$ ample, $K^{2}=9, p_{g}=q=0$. Amer. $J$. Math. 101 (1) (1979), 233-244. Zbl 0433.14021 MR 0527834

[Par] R. Pardini, Abelian covers of algebraic varieties. J. Reine Angew. Math. 417 (1991), 191-213. Zbl 0721.14009 MR 1103912

[Ter] T. Terada, Quelques propriétés géométriques du domaine de $F_{1}$ et le groupe de tresses colorées. Publ. Res. Inst. Math. Sci. 17 (1981), 95-111. Zbl 0466.33010 MR 0613935

Received August 1, 2006

Ingrid C. Bauer, Mathematisches Institut der Universität Bayreuth, Universität Bayreuth, NWII, 95440 Bayreuth, Germany

E-mail: Ingrid.Bauer@uni-bayreuth.de

Fabrizio Catanese, Lehrstuhl Mathematik VIII, Universität Bayreuth, NWII, 95440 Bayreuth, Germany

E-mail: Fabrizio.Catanese@uni-bayreuth.de 